\title{
Ions motion optimization algorithm for multiobjective optimization problems
}

\author{
Hitarth Buch ${ }^{a, b^{*}}$ and Indrajit Trivedi ${ }^{\mathbf{c}}$
}

${ }^{a}$ Research Scholar, Gujarat Technological University, Ahmedabad

${ }^{b}$ Department of Electrical Engineering, Government Engineering College, Rajkot, Gujarat, India

${ }^{c}$ Department of Power Electronics, Vishwakarma Government Engineering College, Chandkheda, Gujarat, India

\begin{tabular}{l}
\hline C H R O N I C L E \\
\hline Article history: \\
Received October 28, 2020 \\
Received in revised format: \\
October 29, 2020 \\
Accepted November 172020 \\
Available online \\
November 17, 2020 \\
\hline Keywords: \\
Multiobjective optimization \\
Ions Motion Algorithm \\
Archive
\end{tabular}

\section{A B S T R A C T}

This paper offers a novel multiobjective approach - Multiobjective Ions Motion Optimization (MOIMO) algorithm stimulated by the movements of ions in nature. The main inspiration behind this approach is the force of attraction and repulsion between anions and cations. A storage and leader selection strategy is combined with the single objective Ions Motion Optimization (IMO) approach to estimate the Pareto optimum front for multiobjective optimization. The proposed method is applied to 18 different benchmark test functions to confirm its efficiency in finding optimal solutions. The outcomes are compared with three novel and well-accepted techniques in the literature using five performance parameters quantitatively and obtained Pareto fronts qualitatively. The comparison proves that MOIMO can approximate Pareto optimal solutions with good convergence and coverage with minimum computational time.

\section{Introduction}

The optimization process looks for finding the minimum or maximum value for single or multiple objectives. Multiobjective optimization refers to optimizing numerous objectives which are often conflicting in nature. Such problems are found in engineering, mathematics, economics, agriculture, politics, information technology, etc. Also, sometimes, the truly optimum solution may not be available at all. In such cases, compromise and estimates are frequently required. Multiobjective optimization is much more complicated than single-objective optimization because of the existence of multiple optimum solutions. At large, all solutions are conflicting, and hence, a group of non-dominated solutions is required to be found out to approximate the true pareto front. Heuristic algorithms are derivative-free solution approaches as they do not use gradient descent to determine the global optimal. Metaheuristic approaches treat the problem as a black box for given inputs and outputs. Problem variables are inputs while objectives are outputs. Many competent metaheuristic approaches were proposed in the past to solve the multiobjective optimization problem. A heuristic approach starts problem optimization by creating an arbitrary group of initial solutions. Every candidate solution is evaluated, objective values are observed, and based on the outputs, the candidate solutions are modified/changed/combined/evolved. This process is continued until the end criteria are met.

There are various difficulties associated while solving the problem using heuristics. Even optimization problems have diverse characteristics. Some of the challenges are constraints, uncertainty, multiple and many objectives, dynamicity. Over a while, global optimum value changes in dynamic problems. Hence, the heuristic approach should be furnished with a suitable operator to keep track of such changes so that the global optimum is not lost. Heuristic approaches should also be fault-tolerant to deal with uncertainty effectively. Constraints divide the search space into viable and unviable solutions. The heuristic approach should be able to discard the unsustainable solution and ultimately discover the best optimum

* Corresponding author.

E-mail address: hitarth buch 020@gtu.edu.in (H. Buch)

(C) 2021 by the authors; licensee Growing Science, Canada. doi: $10.5267 /$ j.dsl.2020.12.001 
solution. Researchers have also proposed surrogate models to reduce computational efforts for computationally expensive functions. The idea of a Pareto dominance operator is introduced to compare more than one objective. The heuristic approach should be able to find all the best Pareto solutions. The proper mechanism should be incorporated with heuristic approaches to deal with multiobjective problems. Archive or storage is necessary to stock the non-dominated solutions found through optimization. Quantity and quality of the best solutions are enhanced by updating the archive residents in every iteration. Another desired characteristic of a multiobjective heuristic approach is to determine several solutions. In other words, the Pareto solutions should binge uniformly across all the objectives.

There are many multiobjective algorithms reported in the literature. The popular algorithms are Multiobjective Particle Swarm Optimization (MOPSO) (Coello 2011), Non-dominated Sorting Genetic Algorithm (NSGA) (Deb et al. 2000; Srinivas and Deb 1994), Multiobjective Ant Colony Optimization (MOALO) (Dorigo and Di Caro 1999), Multi-objective Grey Wolf Optimizer (MOGWO) (S. Mirjalili et al. 2016), Multiobjective Dragonfly Algorithm (MODA) (S. Mirjalili 2016), Multiobjective Multi-Verse Optimizer (MOMVO) (S. Mirjalili, Jangir, et al. 2017), Hybrid Multi-objective Cuckoo Search (HMOCS) (Zhang et al. 2018), Multiobjective Teaching-Learning based Algorithm (Zou et al. 2013), Multiobjective Artificial Bee Colony Algorithm (Akbari et al. 2012), Multiobjective Grasshopper Algorithm (S. Z. S. Mirjalili et al. 2018), Multiobjective Moth Flame Optimization (MOMFO) (Vikas and Nanda 2016), Multiobjective Salp Swarm Optimization (S. Mirjalili, Gandomi, et al. 2017) and Non-dominated Sorting Ions Motion Algorithm (Buch and Trivedi 2020). All these algorithms have proved their efficiency in solving the multiobjective problem. Then a question may arise: Is any new algorithm required still? According to the No Free Lunch (NFL) algorithm (Wolpert and Macready 1997), no algorithm can solve problems of all kinds. Thus, this algorithm allows proposing new algorithms or enhancement of existing ones.

The Ions Motion Algorithm (IMO) (Javidy, Hatamlou, and Mirjalili 2015) has a great exploration with fast convergence speed. The liquid and crystal phase smartly balance the exploration and exploitation stage. These features make the IMO deal with a multiobjective optimization problem potentially. Even the computational difficulty is lesser than several optimization procedures reported in the literature. Such commanding features inspired us to develop a multiobjective version of the existing single objective IMO. In this paper, the proposed algorithm is compared with other well-regarded recent optimization algorithms qualitatively and quantitatively. The remaining paper is arranged thus: Section 2 discusses the multiobjective optimization problem and associated terminology. Section 3 introduces the single objective IMO. Section 4 proposes a novel multiobjective IMO. Findings based on various performance indicators are discussed in Section 5. Section 6 concludes the work and suggests future work.

\section{Introduction to Multiobjective Optimization Problem (MOOP)}

In the single-objective optimization, there is a global optimum unique solution. The reason for this is the presence of only one objective in single-objective optimization problems and the existence of the most excellent unique answer. Evaluation of solutions is simple as there is only one goal and can be completed by the relational operators: $\geq,>, \leq,<$, or $=$. Such problems permit optimization issues to suitably relate the aspirant solutions and ultimately determine the finest one. While in MOOP, though, the answers should be equated with multiple criteria. Multiobjective minimization problem can be stated as follows:

$$
\begin{aligned}
& \min F(\vec{x})=\left\{f_{1}(\vec{x}), f_{2}(\vec{x}), \ldots, f_{n}(\vec{x})\right\} \\
& \text { subject to } \\
& a_{i}(x) \geq 0 \quad i=1,2,3, \ldots, m \\
& b_{i}(x)=0 \quad i=1,2,3, \ldots, p \\
& L_{i b} \leq x_{i} \leq U_{i b} \quad i=1,2,3, \ldots, q
\end{aligned}
$$

Here, $q$ presents a number of variables, $f_{1}(\vec{x}), f_{2}(\vec{x}), \ldots, f_{n}(\vec{x})$ introduces some objective functions, $m$ and $p$ show some inequality and equality constraints. $L_{i b}$ and $U_{i b}$ give lower and upper limits of the variable. Such kind of problems foil us from equating results utilizing the relational operators as there are multiple criteria to evaluate solutions. In a single objective optimization problem, a better solution can be found using a relational operator, but with various objectives, some additional operator(s) is(are) required. The primary operator to equate two solutions bearing in mind multiple objectives is called Pareto optimal dominance and is described as (Carlos A Coello Coello 2009):

The definition I: Pareto Dominance

Let us assume two vectors $\vec{a}=\left(a_{1}, a_{2}, \ldots, a_{k}\right)$ and $\vec{b}=\left(b_{1}, b_{2}, \ldots, b_{k}\right)$ 
Vector $a$ dominates $b$ if and only if:

$$
\forall i \in\{1,2, \ldots, k\}:\left[f_{i}(\vec{a}) \leq f_{i}(\vec{b})\right] \wedge \exists i \in\{1,2, \ldots, k\}:\left[f_{i}(\vec{a}) \prec f_{i}(\vec{b})\right]
$$

By exmining Eq. (5), it may be concluded that one solution is superior to another solution if it has equal, and nonetheless, one improved value in the objectives. Under such a situation, one solution dominates another solution; otherwise, the two solutions are called Pareto optimal or non-dominated solutions. The answers to the multiobjective problem are Pareto optimal solutions. Hence, the Pareto optimality is defined as follows.

Definition II: Pareto optimality (Pareto efficiency) (Ngatchou, Zarei, and El-Sharkawi 2005)

Assuming $a \in A, a$ is Pareto optimal solution if and only if:

$$
\{\nexists \vec{b} \in A \mid \vec{b} \prec \vec{a}\}
$$

Pareto optimality or Pareto efficiency is a state of distribution of solutions from which it is not possible to achieve improvement in any one single objective or preference criterion without deterioration in a specific objective or choice criterion. Such a solution set is known as the Pareto optimal set. The prognosis of the Pareto optimal solutions in the objective search space is called Pareto optimal front.

The definition III: Pareto optimal set (Mirjalili et al., 2017)

The Pareto optimal set comprises a set of Pareto optimal solutions. Mathematically,

$$
P S:=\{\vec{a}, \vec{b} \in A \mid \nexists \vec{b} \prec \vec{a}\}
$$

The definition IV: Pareto optimal front (Mirjalili et al., 2017)

This set consists of objective values for the solutions in the Pareto solutions set. Mathematically, it can be presented as:

$$
\forall i \in\{1,2,3, \ldots, n\}, P F:=\left\{f_{i}(\vec{a}) \mid \vec{a} \in P S\right\}
$$

A quick and easy comparison of solutions of multiobjective optimization can be made with the above four equations. The group of variables, constraints and objectives create a search landscape. Considering the difficulties associated with the representation of search space for the problems with more than one objective, the researchers consider two search spaces: goal and parameter space. Likewise, in single-objective optimization, the range of variables regulates the limits of the search space in each dimension while restraints divulge them.

The overall outlines of all population-based multiobjective algorithms nearly match. They begin the optimization procedure with multiple candidate solutions. Such solutions are equated utilizing the Pareto dominance operator. In every phase of optimization, the repository/storage stores non-dominated solutions and the algorithm attempts to enhance them in the subsequent iteration(s). Different search approaches distinguish one algorithm from another to augment the non-dominated solutions.

\section{Single-objective Ions Motion Algorithm (IMO)}

This section first introduces the single-objective IMO algorithm. The next section presents the multiobjective form of singleobjective IMO.

\subsection{Ions Motion Algorithm}

In 1834, Michael Faraday coined the Greek term 'ion'. Typically, the charged particles are known as ions and can be separated into two categories: cations - ions with positive charge and anions - ions with a negative charge. Fig. 1 presents a conceptual model of force between cations and anions. The primary stimulus of the Ions Motion Algorithm is a force of attraction and repulsion between unlike and like charges, respectively. Javidy et al. (2015) proposed the population-based IMO approach stimulated by these characteristics of ions in nature.

In the IMO algorithm, anions and cations form the candidate solutions for a given optimization problem. The force of attraction/repulsion moves the ions (i.e., candidate solutions) around the search space. The ions are assessed keeping in 
mind the fitness value of the objective function. Anions tend to move towards the best cations while cations tend to move towards the best anions. This movement depends upon the force of attraction/repulsion between them. Such an approach guarantees improvement over iterations but does not guarantee the required exploration and exploitation of search space. Liquid and crystal are two different phases assumed to ensure necessary exploitation and exploration of search space.

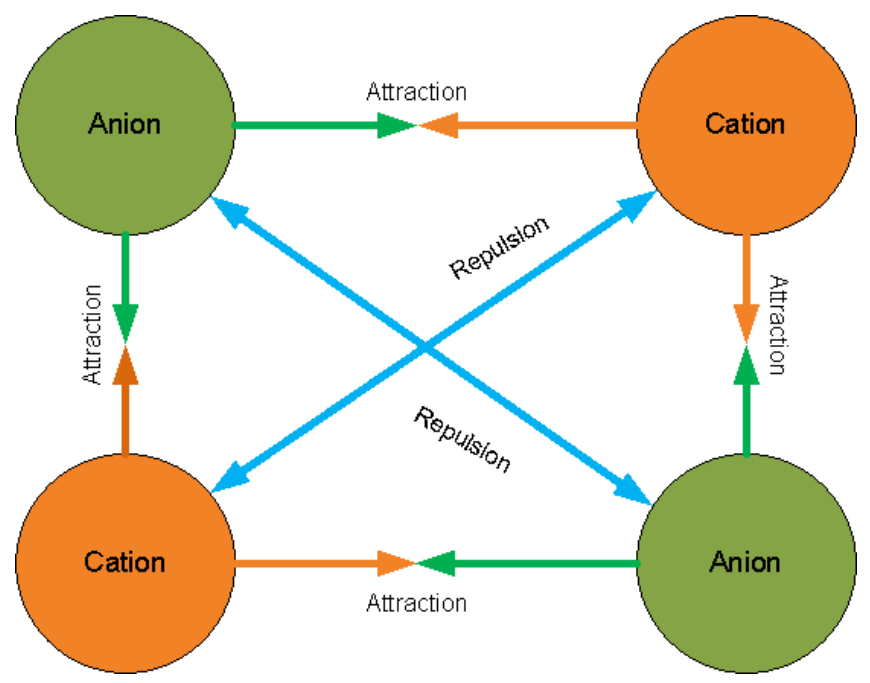

Fig. 1. Conceptual model of the force of attraction and repulsion

\subsubsection{Liquid Phase}

The liquid phase provides more freedom to the movement of ions, and hence, in the liquid stage, the ions can pass quickly. Also, the force of attraction is much more than the force of repulsion. Thus, the force of repulsion can be neglected to explore the search space. The distance between two ions is the only key factor considered to compute the force of attraction. So the resulting mathematical model can be proposed as:

$$
\begin{aligned}
P f_{i, j} & =\frac{1}{1+e^{-0.1 / P d_{i, j}}} \\
Q f_{i, j} & =\frac{1}{1+e^{-0.1 / Q d_{i, j}}}
\end{aligned}
$$

Where $\mathrm{P}$ and $\mathrm{Q}$ present parameters related to anions and cations, respectively. $P d_{i, j}=\left|P_{i, j}-Q b e s t_{j}\right|$ and $Q d_{i, j}=\mid Q_{i, j}-$ Pbest $_{j} \mid . i$ and $j$ present ion index and dimension, respectively. $P d_{i, j}$ is the distance between $i t h$ anion from the best cation in $j$ th dimension, $Q d_{i, j}$ calculates the distance between $i t h$ the cation from the best anion in jth dimension. As presented in Eq. (9) and Eq. (10), force is inversely proportional to distances among ions. Larger the distance, lesser is the force of attraction. In other words, the force of attraction becomes less when the distance grows higher from the best ion with the opposite charge.

According to Eq. (9) and Eq. (10), the value of force varies between 0.5 to $1 . P f_{i, j}$ and $Q f_{i, j}$ are the resultant attraction forces of anions and cations, respectively. After force calculation, the position of positive and negative ions is updated as per the following equations:

$$
\begin{aligned}
& P_{i, j}=P_{i, j}+P f_{i, j}\left(\text { Qbest }_{j}-P_{i, j}\right) \\
& Q_{i, j}=Q_{i, j}+Q f_{i, j}\left(\text { Pbest }_{j}-Q_{i, j}\right)
\end{aligned}
$$

$P f_{i, j}$ and $Q f_{i, j}$ are the resulting attraction forces between opposite ions while $Q b e s t_{j}$ and $P b e s t_{j}$ present the best cations and anions, respectively. The attraction force between ions guarantees exploration. Referring to Eqs. (9-12), the 
conclusion can be drawn that in the liquid phase, there is no involvement of the random component. Fig. 2 presents an abstract model of the movement of ions in the liquid stage. With an increasing number of iterations, more and more ions start interacting, converging towards the best ion with an opposite charge, and hence, exploration gradually decreases. This phenomenon is precisely like a conversion from liquid to crystal state observed in nature. The search agents, i.e., ions, also enter crystal state, finally converging towards the best solution in search space.

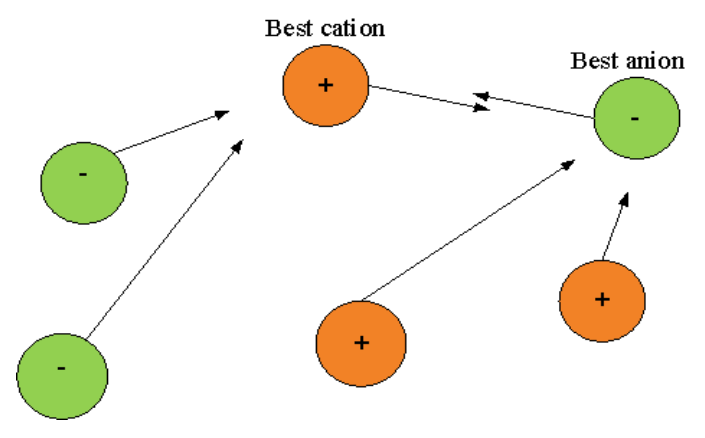

Fig. 2. Ions movement towards the best ions in the liquid phase

\subsubsection{Crystal Phase}

In this stage, the ions congregate to the optimal solution. Convergence has already taken place. Since the search space has an unknown form, occasionally convergence gets trapped into local minima. A separate mechanism is proposed at the crystal stage to avoid trapping of solutions in local minima. The cations and anions in the crystal phase are organized to maximize their force of attraction. When an outside force is applied to the same charges in the crystal phase, the resultant repulsion force cracks the crystal apart. Mathematically, the mechanism to overcome local optimum trapping can be demonstrated as below:

if (QbestFit $\geq$ QworstFit/2 and PbestFit $\geq$ PworstFit/2)

$$
\begin{aligned}
& \quad \text { if rand }()>0.5 \\
& \quad P_{i}=P_{i}+\varphi_{1} \times(\text { Qbest }-1) \\
& \text { else } \\
& P_{i}=P_{i}+\varphi_{1} \times(\text { Qbest }) \\
& \text { end if } \\
& \text { if rand }()>0.5 \\
& \quad Q_{i}=Q_{i}+\varphi_{2} \times(\text { Pbest }-1)
\end{aligned}
$$

$$
\begin{aligned}
& \text { else } \\
& \qquad Q_{i}=Q_{i}+\varphi_{2} \times(\text { Pbest }) \\
& \text { end if } \\
& \text { if rand }()<0.5 \\
& \quad \operatorname{Re} \text {-initialize } \mathrm{P}_{\mathrm{i}} \text { and } \mathrm{Q}_{\mathrm{i}} \\
& \text { end if }
\end{aligned}
$$

\section{end if}

Where, $\varphi_{1}$ and $\varphi_{2}$ are random numbers between $[-1,1]$ and $\operatorname{rand}()$ is a random number between $[0,1]$. QbestFit and QworstFit present fitness of the best and worst cations. PbestFit and PworstFit are the fitness of best and worst anions. The best fitness of anions and cations should be better than or equal to the average competence of the worst anions and cations. If this situation is met, ions are arbitrarily navigated in search space to circumvent stagnation adjacent to local minima. Again, ions enter the liquid state until termination criteria are met.

It should be noticed here that in Eq. (13), four, instead of two, conditions are proposed to achieve different behavior of the proposed algorithm. These four conditions are presented below: 
1. Both first if-else statements are met:

$$
\begin{aligned}
& P_{i}=P_{i}+\varphi_{1} \times(\text { Qbest }-1) \\
& Q_{i}=Q_{i}+\varphi_{2} \times(\text { Pbest }-1)
\end{aligned}
$$

2. Only the first if-else statement is met:

$$
\begin{aligned}
& P_{i}=P_{i}+\varphi_{1} \times(\text { Qbest }-1) \\
& Q_{i}=Q_{i}+\varphi_{2} \times(\text { Pbest })
\end{aligned}
$$

3. Only the second if-else statement is met:

$$
\begin{aligned}
& P_{i}=P_{i}+\varphi_{1} \times(\text { Qbest }) \\
& Q_{i}=Q_{i}+\varphi_{2} \times(\text { Pbest }-1)
\end{aligned}
$$

4. Both conditions are not met:

$$
\begin{aligned}
& P_{i}=P_{i}+\varphi_{1} \times(\text { Qbest }) \\
& Q_{i}=Q_{i}+\varphi_{2} \times(\text { Pbest })
\end{aligned}
$$

In contrast, merging the first two if-else statements will result in only two conceivable combinations:

5. Collective if-else sentences are fulfilled:

$$
\begin{aligned}
& P_{i}=P_{i}+\varphi_{1} \times(\text { Qbest }-1) \\
& Q_{i}=Q_{i}+\varphi_{2} \times(\text { Pbest }-1)
\end{aligned}
$$

6. Combined if-else sentences are not fulfilled:

$$
\begin{aligned}
& P_{i}=P_{i}+\varphi_{1} \times(\text { Qbest }) \\
& Q_{i}=Q_{i}+\varphi_{2} \times(\text { Pbest })
\end{aligned}
$$

Thus, splitting two conditions into four provide different behavior for the IMO which helps avoid local optimal entrapment. Fig. 3 presents the standard steps of the Ions Motion Algorithm. The IMO starts with a random group of solutions. The arbitrary collection of solutions during initialization is generated using $r\left(u b_{i}-l b_{i}\right)+l b_{i}$ where $r$ is a random number with uniform distribution in the interval $(0,1) \cdot u b_{i}$ and $l b_{i}$ represent upper and lower bound respectively of $i$ th variable. At this phase, ions are equally separated into a set of anions and cations, respectively. The fitness of each anion and cation is calculated, and according to fitness, the best and worst anions/cations are selected and saved. The attraction forces and positions are updated using Eqs. (9-12). During each iteration, if the condition of the crystal phase is met, the ions go into the crystal phase. Till the satisfaction of termination criteria, ions keep going between solid and liquid phases. In the end, the best ion is reported as the best approximation of the global solution.

\section{Multiobjective Ions Motion Algorithm (MOIMO)}

The single objective IMO algorithm can drive ions towards the best ion and update it throughout the iterations. But, this approach cannot solve multiobjective problems mainly owing to the following two reasons:

- $\quad$ IMO cannot store multiple best solutions as it preserves a unique solution as the optimum solution.

- IMO updates the ion position concerning the unique best solution attained up to now during each iteration, but MOOPs have no single best solution.

The first limitation is handled by incorporating the IMO algorithm with the storage of ions. This storage preserves the best non-dominated solutions attained so far throughout optimization and is very close to the archive (storage) mechanism employed in MOPSO ( Coello \& Lechuga 2002) and Pareto Archived Evolution Strategy (PAES) (Knowles and Corne 1999). The storage has the maximum storage capacity to stock the limited quantity of solutions. Throughout optimization, every ion is compared with every storage element using Pareto dominance operators. Such a comparison may provide us following different cases: 


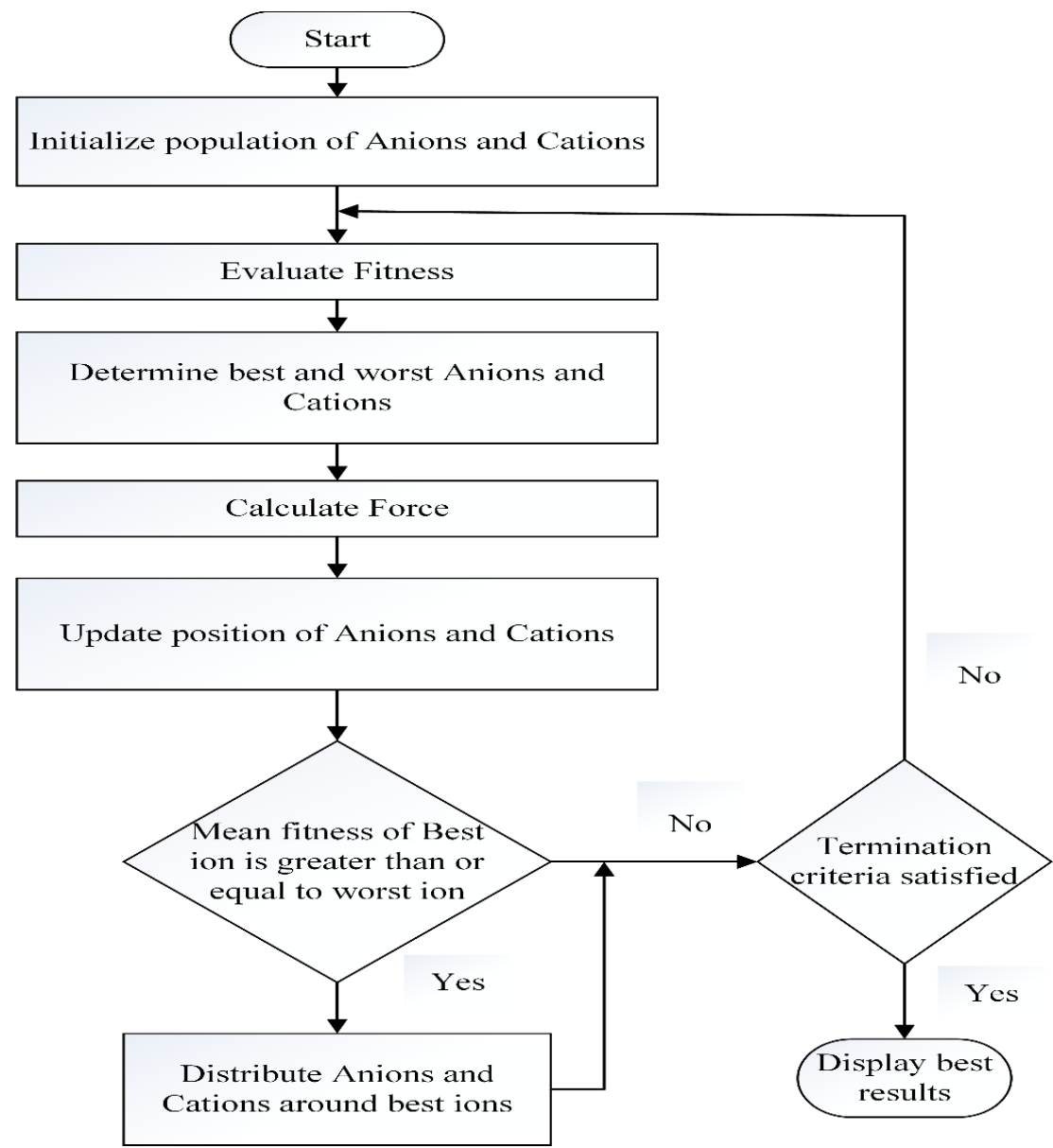

Fig. 3. The standard procedure of the IMO algorithm

1. If the new solution dominates archive residents, then they should be swapped.

2. If the new solution leads a group of solutions in the storage, they all should be deleted from the storage, and a new solution should be added in the storage.

3. If the storage is full and the new solution is non-dominated in comparison with the storage elements, then the most straightforward means is to remove any solution randomly and swap it with the new non-dominated solution. However, as the apriori method should be able to determine uniformly dispersed Pareto front, the best candidate to be eliminated from the storage is the one in the densest area. Such a technique will improve the distribution of repository residents throughout the iterations.

The number of neighboring solutions with a specified maximum distance is counted and assumed to find the non-dominated solution(s) with a populated neighborhood. This distance is calculated by $d=\frac{\max -\min }{\text { repository size }}$ where max and $\min$ are two vectors for storing maximum and minimum objective values, respectively. The storage with one solution in each segment is an ideal case. Based on the number of neighboring repository residents, each solution is assigned a rank. After assigning a rank to every storage element depending upon the number of neighboring solutions, a roulette wheel mechanism is employed to choose one of them. Higher the rank of a solution, higher the likelihood of eliminating it from the storage.

The second component is a leader selection strategy that assists in choosing the best ions as there are multiple best solutions in a multiobjective search space. Again, the ion can be selected arbitrarily from the storage. However, a more suitable method is to choose an answer from the least populated region. A similar mechanism is applied using the roulette wheel selection and the same ranking process. The main difference between archive maintenance and leader selection is that in archive maintenance, the solution is likely to be chosen from the crowded neighborhood while in contrast, for leader selection strategy, the solution is expected to be selected from a less crowded area. Fig. 4 presents a schematic representation of an archive-based plan for a multiobjective optimization approach.

In a nutshell, the MOIMO approach first generates the population of ions concerning the upper and lower bounds of variables. This approach then computes the objective values of each ion and determines the non-dominated ones. If the 
storage has a vacancy, then the non-dominated solutions are added into it. If the storage is completely occupied, the archive maintenance is run to remove the solution with a crowded neighborhood. In this case, the solutions are ranked and chosen through a roulette wheel. After eliminating the required number of solutions from the storage, the new solutions are added to the storage. After updating the storage, an ion is selected from the non-dominated solutions having the least crowded neighborhood. The next step is to update the positions. All the above steps are repeated (except initialization), till the termination criteria are met.
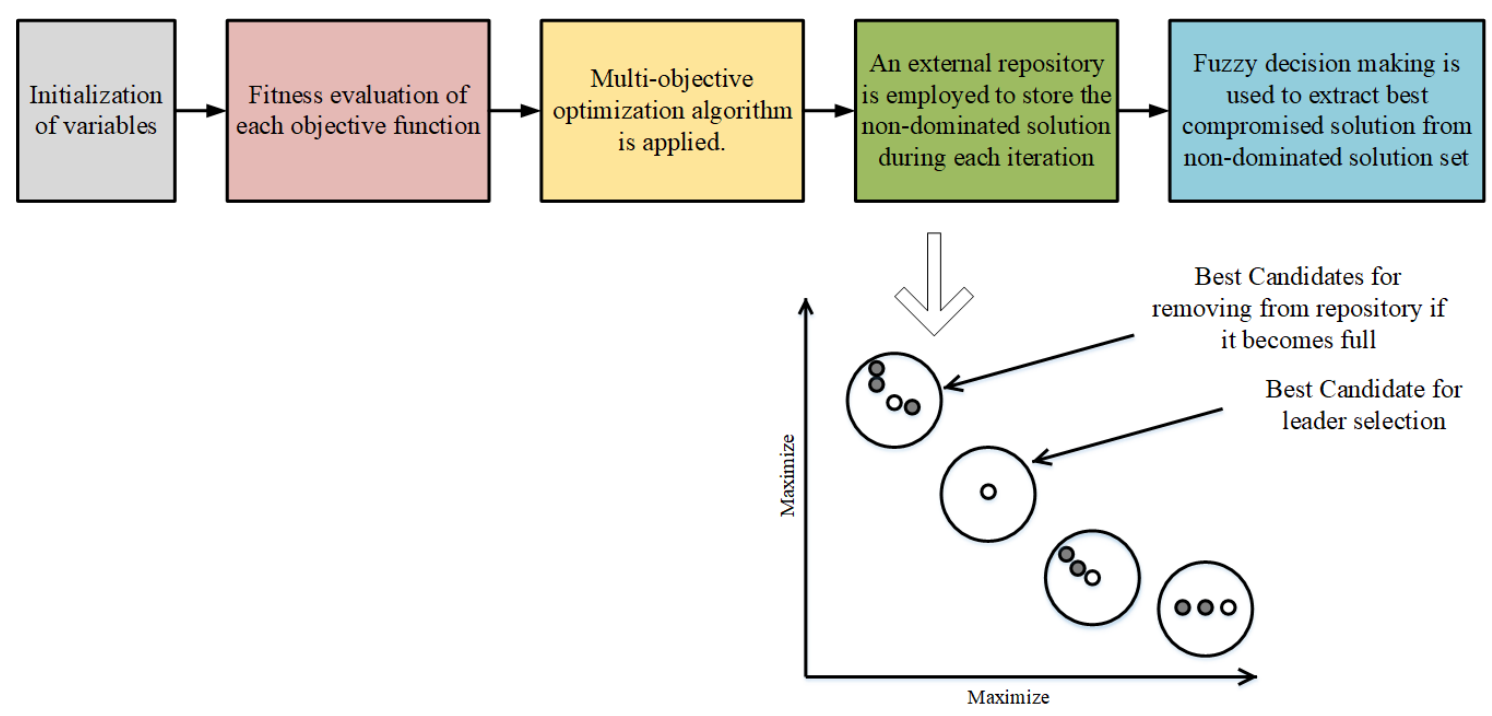

Fig. 4. Schematic representation of archive-based multiobjective optimization algorithm

The following vital observations can be made regarding the MOIMO algorithm:

1. The non-dominated solutions attained are deposited in storage, so they never get lost even if the whole population worsens during an iteration.

2. The solutions with the dense neighborhood are rejected whenever storage maintenance is called. This results in refining the diversity of non-dominated solutions across all objectives.

3. The best anion and cation are selected from the list of repository members having the smallest number of neighboring solutions which concentrates the search to the less dense regions of the Pareto front and thus enhances the diversification.

4. MOIMO inherits the search mechanism from basic IMO.

The computational complexity of $\mathrm{MOIMO}$ is $\mathrm{O}\left(\mathrm{PQ}^{2}\right)$, where $\mathrm{P}$ and $\mathrm{Q}$ are numbers of objectives and solutions, respectively. Thus, computational complexity is comparable to PAES (Knowles and Corne 1999) and MOPSO (C A Coello Coello and Lechuga 2002). With the procedure mentioned above, MOIMO can find Pareto optimal solutions, store them in the repository and enhance their dispersal. The next section discusses the performance of MOIMO on the standard benchmark functions.

\section{Findings on Test Functions}

\subsection{Experimental Setup}

Various benchmark test functions having diverse characteristics are employed to scrutinize the performance of multiobjective optimization algorithms as different test functions can challenge an algorithm from different standpoints. The benchmark functions used are (Mirjalili et al., 2016):

- Unconstrained multiobjective test functions (ZDT1, ZDT2, ZDT3, ZDT4, ZDT6, SCH1, SCH2)

- Constrained multiobjective test functions (BEL, BNH, CONSTR, CF1, OSY)

- Engineering design multiobjective problems (3 Bar Truss, Brushless DC Wheel Motor, Pressure Vessel, Satellite Pipe, Welded Beam, Car Crash Design Problem)

The performance of MOIMO is compared with other well-regarded algorithms in literature like MOMVO (S. Mirjalili, Jangir, et al. 2017), MODA (S. Mirjalili 2016), and MOALO (S. Mirjalili, Jangir, and Saremi 2017). The results are evaluated quantitatively and qualitatively. For qualitative comparison, the best Pareto optimal front over ten independent runs is chosen. However, the qualitative comparison does not indicate how much an algorithm is better than the other one. 
And hence, different quantitative performance indicators are employed. The first metric is the Generational Distance (GD) (Van Veldhuizen 1999). GD is calculated between the true Pareto front and obtained the Pareto front. It calculates the average distance between the obtained Pareto front and the true Pareto front and can be mathematically presented as:

$$
G D=\frac{\left(\sum_{p=1}^{k}\left(d_{i}\right)\right)}{k}
$$

where,

$$
d_{i}=\left(\sum_{i=1}^{n}\left(P F_{i . p}^{o}-P F_{i . p}^{t}\right)^{2}\right)^{1 / 2}
$$

Here $k$ is the number of Pareto solutions, $n$ is the number of objective functions, $P F_{i . p}^{o}$ indicate the $p^{t h}$ obtained Pareto solution for the ith objective, and $P F_{i . p}^{t}$ indicate the nearest point on the true Pareto front from $P F_{i . p}^{o}$.
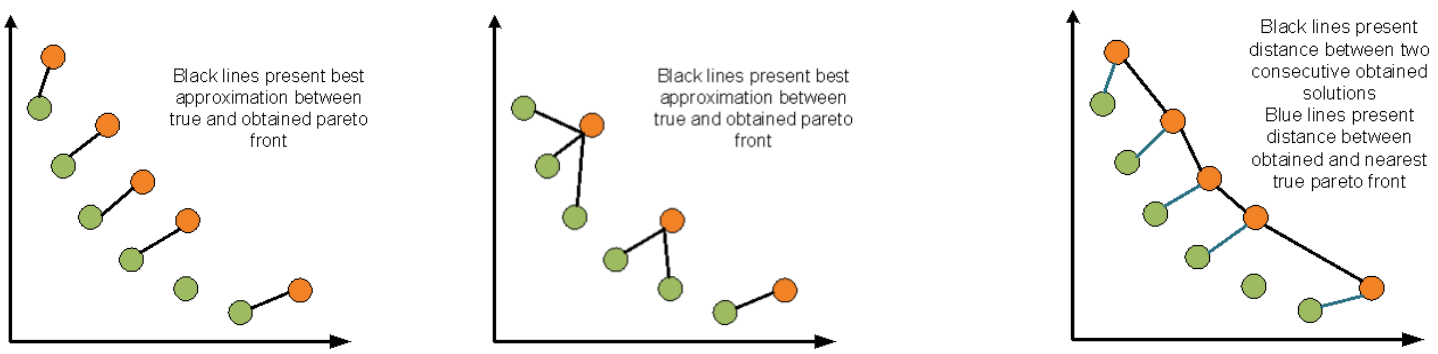

Fig. 5. Schematic representation of (a) Generational Distance (GD) (b) Inverse Generational Distance (IGD) and (c) spread

Another indicator used is Inverse Generational Distance (IGD) (Coello and Cortés 2005). It calculates the distance between each element of the obtained front from the true Pareto front keeping true Pareto front as a reference. It is mathematically defined as:

$$
I G D=\frac{\sqrt{\sum_{i=1}^{Q} d_{i}^{2}}}{Q}
$$

where $Q$ presents the number of solutions in the true Pareto front and $d_{i}$ is the Euclidean distance between each of the solutions in true Pareto front and the nearest member from the set of non-dominated solutions found by the algorithm. The IGD measures both the diversity and the convergence of an obtained non-dominated solution set. The smaller value of IGD presents closeness between true and obtained Pareto front.

Spread (Deb, 2001) and spacing (S) (Schott, 1995) help measure coverage. Coverage helps identify the distribution of obtained solutions over true Pareto front. Mathematically, the spread is defined as:

$$
\Delta=\frac{\sum_{j=1}^{n} d_{j}^{e x}+\sum_{p=2}^{k}\left|d_{p}-\bar{d}\right|}{\sum_{j=1}^{n} d_{j}^{e x}+(k-1) \bar{d}}
$$

Here, $d_{p}$ is the Euclidian distance between two consecutive points on the obtained Pareto front and $d_{j}^{e x}$ is the Euclidian distance between the obtained Pareto front and true Pareto front. $\bar{d}$ is the average of $d_{p}$. Spread verifies the condition for the obtained Pareto front to cover the true Pareto front. Smaller the value of spread, better the spread, i.e., the uniform distribution of obtained solutions. Another indicator, i.e., the spacing is defined as: 


$$
S=\frac{1}{k-1} \sum_{p=2}^{k}\left(D_{p}-\bar{D}\right)^{2}
$$

Here, $D_{p}$ is the absolute difference between two consecutive solutions in the obtained Pareto front. It is defined as:

$$
D_{p}=\sum_{i}^{n}\left|P F_{i \cdot(p-1)}^{0}-P F_{i . p}^{0}\right|
$$

where, $\bar{D}$ is the average of all $D_{p}$. Spacing specifies the spread of the obtained Pareto front. The higher value of $S$ indicates the better coverage of the obtained Pareto solutions. Fig. 5. schematically represents the generational distance, inverse generational distance and spread. Each algorithm is run for 10 independent times for 200 iterations each. The archive size and the number of search agents are set to 100 for constrained and unconstrained functions while they are set to 200 for real-world benchmark functions. For a fair comparison, the population size is set to 100 for constrained and unconstrained benchmark functions. For real-world engineering design problems, the population size is set to 200 . The results are tabulated in the next subsections and the best values are highlighted in bold.

\subsection{Results on Unconstrained Test Functions}

The first set of test problems contain unconstrained standard benchmark functions. Seven different benchmark functions, i.e., ZDT1, ZDT2, ZDT3, ZDT4, ZDT6, SCHN1 and SCHN2 are employed to check the performance of the MOIMO. Fig. 6. and Table 1 to Table 4 present qualitative and quantitative performance assessment for the unconstrained test functions. As shown in Fig. 6. , for all the unconstrained test functions, MOIMO provides better coverage as compared to the rest of the algorithms. In one of the most challenging test functions, i.e., ZDT3, MOIMO provides uniform coverage in all the algorithms.

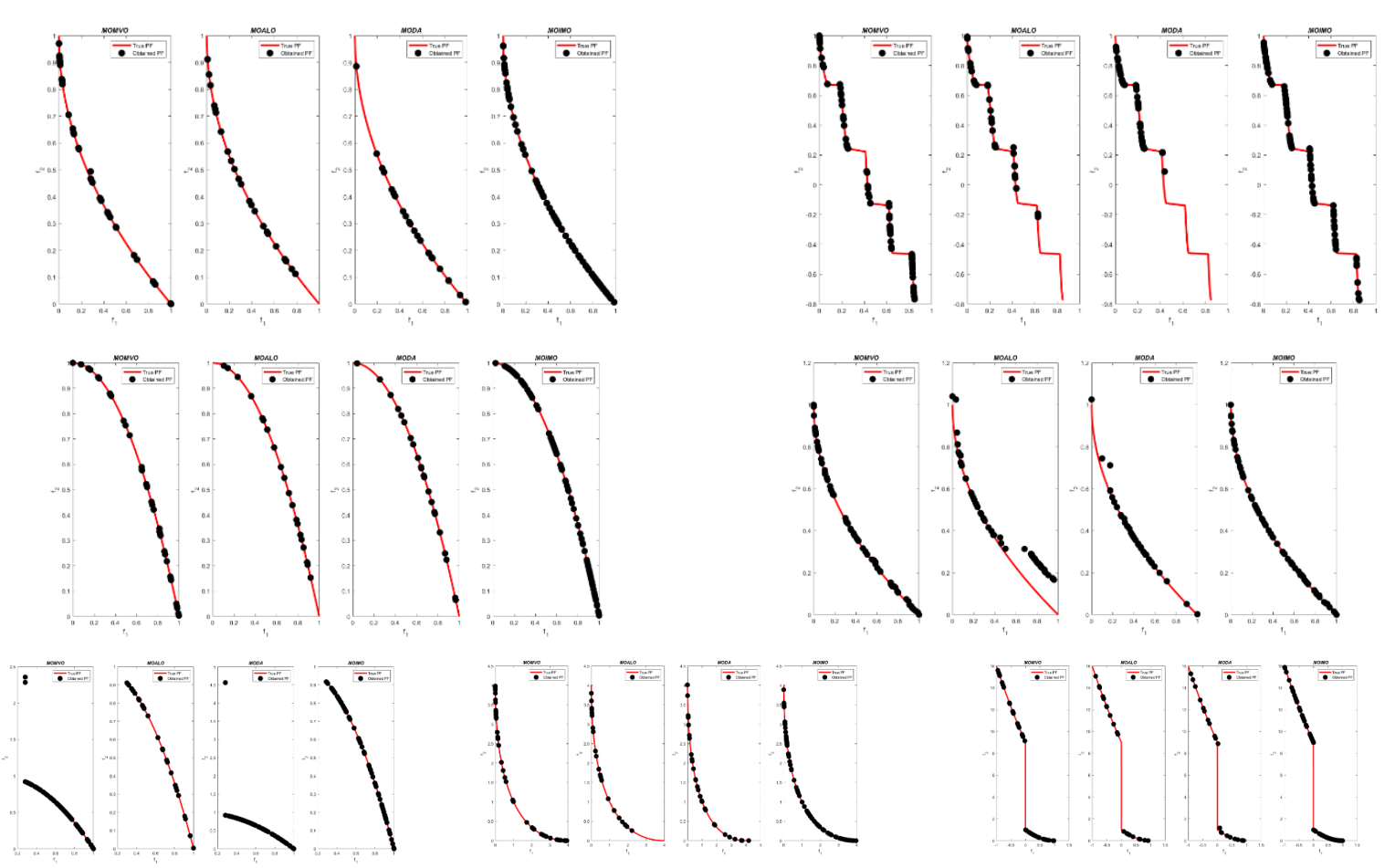

Fig. 6. Best Pareto front for unconstrained benchmark functions

While analyzing the results quantitatively, it is seen that, for most performance indicators, the MOIMO provides significantly better results than the rest of the algorithms. MOIMO is compared for the average value of each performance indicator and standard deviation obtained over 10 independent runs. These results display the superiority of MOIMO showing higher accuracy and better robustness. Thus, MOIMO has the potential to outclass the rest of the algorithms in achieving Pareto optimal front with non-convex non-uniform regions.

Table 1

Results of the multiobjective algorithm (using GD) on the unconstrained test functions 


\begin{tabular}{|c|c|c|c|c|}
\hline & MOMVO & MOALO & MODA & MOIMO \\
\hline \multicolumn{5}{|l|}{ ZDT1 } \\
\hline Avg. GD & 0.0065457 & 0.00010694 & $9.4009 \mathrm{e}-05$ & 0.00015758 \\
\hline $\mathrm{SD}$ of GD & 0.013886 & $2.207 \mathrm{e}-05$ & $3.6339 \mathrm{e}-05$ & $7.1257 \mathrm{e}-05$ \\
\hline \multicolumn{5}{|l|}{ ZDT2 } \\
\hline Avg. GD & 0.017519 & $8.2369 \mathrm{e}-05$ & 0.0018219 & $9.6513 \mathrm{e}-05$ \\
\hline SD of GD & 0.038833 & $3.3481 \mathrm{e}-05$ & 0.0038699 & $8.5783 \mathrm{e}-06$ \\
\hline \multicolumn{5}{|l|}{ ZDT3 } \\
\hline Avg. GD & 0.00045131 & 0.00059499 & 0.00051111 & 0.00031746 \\
\hline $\mathrm{SD}$ of GD & $4.0207 \mathrm{e}-05$ & 0.00016385 & 0.0003006 & $6.1964 \mathrm{e}-06$ \\
\hline \multicolumn{5}{|l|}{ ZDT4 } \\
\hline Avg. GD & 0.0004134 & 0.0075542 & 0.0029169 & 0.00031644 \\
\hline $\mathrm{SD}$ of GD & 0.00012338 & 0.006953 & 0.0041258 & $8.5969 \mathrm{e}-05$ \\
\hline \multicolumn{5}{|l|}{ ZDT6 } \\
\hline Avg. GD & 0.016918 & 0.073206 & 0.08976 & $8.7355 \mathrm{e}-05$ \\
\hline $\mathrm{SD}$ of GD & 0.011282 & 0.087903 & 0.14623 & $4.2786 \mathrm{e}-06$ \\
\hline \multicolumn{5}{|l|}{$\mathrm{SCH} 1$} \\
\hline $\mathrm{SD}$ of GD & $1.913 \mathrm{e}-05$ & $3.2288 \mathrm{e}-05$ & 0.00012406 & $1.0351 \mathrm{e}-05$ \\
\hline Avg. GD & 0.00024794 & 0.00024983 & 0.00033026 & 0.00023967 \\
\hline \multicolumn{5}{|l|}{$\mathrm{SCH} 2$} \\
\hline $\mathrm{SD}$ of GD & $2.5767 \mathrm{e}-05$ & $2.301 \mathrm{e}-05$ & 0.00015192 & 0.0025352 \\
\hline Avg. GD & $1.786 \mathrm{e}-06$ & $2.6257 \mathrm{e}-06$ & 0.00015659 & 0.00073378 \\
\hline
\end{tabular}

Table 2

Results of the multiobjective algorithm (using IGD) on the unconstrained test functions

\begin{tabular}{|c|c|c|c|c|}
\hline & MOMVO & MOALO & MODA & MOIMO \\
\hline \multicolumn{5}{|l|}{ ZDT1 } \\
\hline Avg. IGD & 0.0065457 & 0.00010694 & 0.0018231 & 0.00054853 \\
\hline SD of IGD & 0.013886 & $2.207 \mathrm{e}-05$ & 0.00055216 & $9.422 \mathrm{e}-05$ \\
\hline \multicolumn{5}{|l|}{ ZDT2 } \\
\hline Avg. IGD & 0.0014645 & 0.0019369 & 0.0021012 & 0.00064484 \\
\hline SD of IGD & 0.00013103 & 0.00061345 & 0.0015287 & $8.5653 e-05$ \\
\hline \multicolumn{5}{|l|}{ ZDT3 } \\
\hline Avg. IGD & 0.00045131 & 0.00059499 & 0.00051111 & 0.00068141 \\
\hline SD of IGD & $4.0207 \mathrm{e}-05$ & 0.00016385 & 0.0003006 & 0.0001457 \\
\hline \multicolumn{5}{|l|}{ ZDT4 } \\
\hline Avg. IGD & 0.0022795 & 0.0030114 & 0.0028121 & 0.00053503 \\
\hline SD of IGD & 0.0032244 & 0.00089163 & 0.0017037 & $5.5694 \mathrm{e}-05$ \\
\hline \multicolumn{5}{|l|}{ ZDT6 } \\
\hline Avg. IGD & 0.00054492 & 0.0017166 & 0.0086791 & 0.00057224 \\
\hline SD of IGD & 0.00010866 & 0.0010588 & 0.016899 & $4.4493 e-05$ \\
\hline \multicolumn{5}{|l|}{$\mathrm{SCH} 1$} \\
\hline SD of IGD & 0.002219 & 0.003891 & 0.0018651 & 0.0010189 \\
\hline Avg. IGD & 0.0011036 & 0.0030438 & 0.00030546 & 0.0002428 \\
\hline \multicolumn{5}{|l|}{$\mathrm{SCH} 2$} \\
\hline SD of IGD & 0.00054056 & 0.00080233 & 0.0007836 & 0.00025411 \\
\hline Avg. IGD & $7.0394 \mathrm{e}-05$ & 0.00015486 & 0.00012158 & $3.2892 \mathrm{e}-05$ \\
\hline
\end{tabular}

Table 3

Results of the multiobjective algorithm (using spread) on the unconstrained test functions

\begin{tabular}{|c|c|c|c|c|}
\hline & MOMVO & MOALO & MODA & MOIMO \\
\hline \multicolumn{5}{|l|}{ ZDT1 } \\
\hline Avg. Spread & 1.4095 & 1.4769 & 1.4839 & 0.89417 \\
\hline SD of Spread & 0.038734 & 0.038021 & 0.1671 & 0.1075 \\
\hline \multicolumn{5}{|l|}{ ZDT2 } \\
\hline Avg. Spread & 1.4492 & 1.4704 & 1.4719 & 0.85165 \\
\hline SD of Spread & 0.049026 & 0.026366 & 0.083441 & 0.035557 \\
\hline \multicolumn{5}{|l|}{ ZDT3 } \\
\hline Avg. Spread & 1.1088 & 1.363 & 1.3287 & 1.0253 \\
\hline SD of Spread & 0.055513 & 0.043786 & 0.17185 & 0.076027 \\
\hline \multicolumn{5}{|l|}{ ZDT4 } \\
\hline Avg. Spread & 0.88654 & 1.2407 & 1.3232 & 0.84776 \\
\hline SD of Spread & 0.058543 & 0.074618 & 0.10319 & 0.056613 \\
\hline \multicolumn{5}{|l|}{ ZDT6 } \\
\hline Avg. Spread & 1.075 & 1.3359 & 1.3769 & 0.90682 \\
\hline SD of Spread & 0.07035 & 0.091983 & 0.17152 & 0.038463 \\
\hline \multicolumn{5}{|l|}{ SCH1 } \\
\hline SD of Spread & 1.2704 & 1.5001 & 1.4519 & 0.87412 \\
\hline Avg. Spread & 0.065065 & 0.059148 & 0.046144 & 0.044177 \\
\hline \multicolumn{5}{|l|}{$\mathrm{SCH} 2$} \\
\hline SD of Spread & 1.3494 & 1.6203 & 1.5984 & 1.001 \\
\hline Avg. Spread & 0.070502 & 0.074842 & 0.0639 & 0.028742 \\
\hline
\end{tabular}

\section{Table 4}

Results of the multiobjective algorithm (using spacing) on the unconstrained test functions 


\begin{tabular}{|c|c|c|c|c|}
\hline & MOMVO & MOALO & MODA & MOIMO \\
\hline \multicolumn{5}{|l|}{ ZDT1 } \\
\hline Avg. Spacing & 0.088253 & 0.012002 & 0.013515 & 0.057615 \\
\hline SD of Spacing & 0.12858 & 0.0086463 & 0.0059354 & 0.0083665 \\
\hline \multicolumn{5}{|l|}{ ZDT2 } \\
\hline Avg. Spacing & 0.18524 & 0.016203 & 0.035237 & 0.055014 \\
\hline SD of Spacing & 0.38283 & 0.0042029 & 0.046016 & 0.014261 \\
\hline \multicolumn{5}{|l|}{ ZDT3 } \\
\hline Avg. Spacing & 0.12386 & 0.03244 & 0.049203 & 0.13544 \\
\hline SD of Spacing & 0.030695 & 0.015926 & 0.03728 & 0.027096 \\
\hline \multicolumn{5}{|l|}{ ZDT4 } \\
\hline Avg. Spacing & 0.044365 & 0.044564 & 0.040731 & 0.052348 \\
\hline SD of Spacing & 0.0044424 & 0.046192 & 0.039918 & 0.010145 \\
\hline \multicolumn{5}{|l|}{ ZDT6 } \\
\hline Avg. Spacing & 0.19458 & 0.15945 & 0.23784 & 0.071907 \\
\hline SD of Spacing & 0.096783 & 0.10851 & 0.22936 & 0.0080407 \\
\hline \multicolumn{5}{|l|}{ SCH1 } \\
\hline Avg. of Spacing & 0.19661 & 0.082656 & 0.19319 & 0.46286 \\
\hline SD of Spacing & 0.096635 & 0.041882 & 0.068511 & 0.067796 \\
\hline \multicolumn{5}{|l|}{$\mathrm{SCH} 2$} \\
\hline Avg. of Spacing & 1.472 & 0.071143 & 0.29546 & 2.0499 \\
\hline SD of Spacing & 0.41896 & 0.094253 & 0.39373 & 0.87483 \\
\hline
\end{tabular}

\subsection{Results on Constrained Test Functions}

The next group of test function comprises five constrained benchmark functions. We must include the constraint handling method with MOIMO to make it capable of solving such problems. Identifying an appropriate constraint handling method is out of the scope. In this work, a death penalty function (Carlos, 2000) is used to punish search agents that violate any of the constraints at any stage. For equating algorithms, four metrics are applied in this research: GD, IGD, metric of spread and metric of space. Table 5 to Table 8 present quantitative results. These performance pointers permit us to enumerate and equate algorithms regarding diversification and intensification. Fig. 3 presents the shape of the best Pareto front achieved by the four algorithms on constrained benchmark functions. Reviewing these figures, MODA presents poor performance, notwithstanding its good coverage in a few cases. However, MOIMO and MOMVO both offer a better convergence close to all true Pareto fronts.
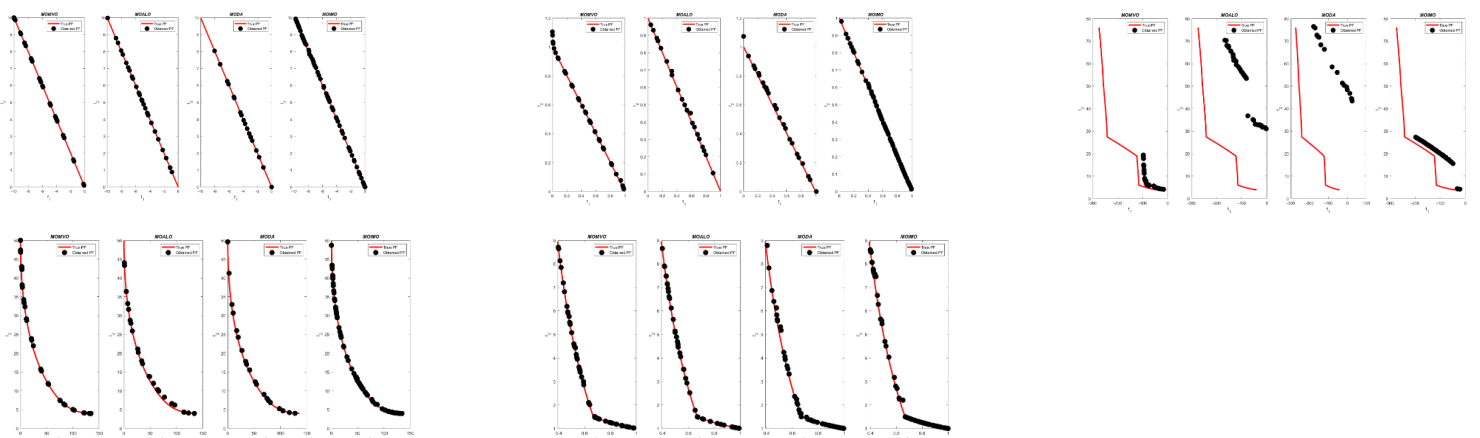

Fig. 3. Best pareto front for constrained benchmark functions

Table 5

Results of the multiobjective algorithm (using GD) on the constrained test functions

\begin{tabular}{|c|c|c|c|c|}
\hline & MOMVO & MOALO & MODA & MOIMO \\
\hline \multicolumn{5}{|l|}{ BEL } \\
\hline Avg. GD & $7.4819 \mathrm{e}-05$ & 0.00022544 & 0.0001356 & 0.00023102 \\
\hline SD of GD & $4.8942 \mathrm{e}-05$ & 0.00026187 & 0.0001369 & 0.00026533 \\
\hline \multicolumn{5}{|l|}{$\mathrm{BNH}$} \\
\hline Avg. GD & 0.0022643 & 0.00094086 & 0.00061468 & 0.00039748 \\
\hline SD of GD & 0.00032499 & 0.00030889 & 0.0003078 & $3.5912 \mathrm{e}-05$ \\
\hline \multicolumn{5}{|l|}{$\mathrm{CF} 1$} \\
\hline Avg. GD & 0.005943 & 0.002448 & 0.002289 & 0.0020175 \\
\hline SD of GD & 0.0010235 & 0.0001741 & 0.0002851 & $7.7036 e-05$ \\
\hline \multicolumn{5}{|l|}{ CONSTR } \\
\hline Avg. GD & 0.00033082 & 0.00087881 & 0.0012952 & 0.00072863 \\
\hline SD of GD & $4.0943 \mathrm{e}-05$ & 0.00062172 & 0.00058203 & 0.00013165 \\
\hline \multicolumn{5}{|l|}{ OSY } \\
\hline Avg. GD & 0.0078333 & 0.027875 & 0.04946 & 0.011565 \\
\hline SD of GD & 0.00093499 & 0.018849 & 0.012052 & 0.0023181 \\
\hline
\end{tabular}

Table 6 
Results of the multiobjective algorithm (using IGD) on the constrained test functions

\begin{tabular}{lllll}
\hline & MOMVO & MOALO & MODA & MOIMO \\
\hline BEL & & & \\
Avg. IGD & 0.0011629 & 0.0012543 & 0.0018785 & $\mathbf{0 . 0 0 0 3 9 7 5 1}$ \\
SD of IGD & 0.0001322 & 0.00030576 & 0.00082551 & $\mathbf{2 . 8 1 2 e - 0 5}$ \\
BNH & & & 0.0032425 & $\mathbf{0 . 0 0 1 0 1 5 3}$ \\
Avg. IGD & 0.0022643 & 0.0030569 & 0.0011784 & $\mathbf{0 . 0 0 0 1 0 6 9 9}$ \\
SD of IGD & 0.00032499 & 0.00073384 & & \\
CF1 & & & 0.0069698 & $\mathbf{0 . 0 0 2 6 3 6 5}$ \\
Avg. IGD & 0.005943 & 0.0084038 & 0.001894 & $\mathbf{0 . 0 0 0 6 1 8 0 7}$ \\
SD of IGD & 0.0010235 & 0.00089992 & 0.0011423 & $\mathbf{0 . 0 0 0 8 1 5 8 9}$ \\
CONSTR & 0.00087387 & 0.0010705 & 0.00040386 & 0.00026318 \\
Avg. IGD & 0.00014332 & $\mathbf{0 . 0 0 0 1 4 3 1 4}$ & 0.011433 & \\
SD of IGD & & 0.0029412 & $\mathbf{0 . 0 0 7 0 9 7 5}$ \\
OSY & 0.010584 & 0.0095221 & & $\mathbf{0 . 0 0 1 6 0 2 2}$ \\
Avg. IGD & 0.0044182 & 0.0016712 & & \\
SD of IGD & & & \\
\hline
\end{tabular}

Table 7

Results of the multiobjective algorithm (using spread) on the constrained test functions

\begin{tabular}{|c|c|c|c|c|}
\hline & MOMVO & MOALO & MODA & MOIMO \\
\hline \multicolumn{5}{|l|}{ BEL } \\
\hline Avg. Spread & 1.5036 & 1.4448 & 1.5583 & 0.92292 \\
\hline SD of Spread & 0.023962 & 0.058427 & 0.095943 & 0.063347 \\
\hline \multicolumn{5}{|l|}{$\mathrm{BNH}$} \\
\hline Avg. Spread & 1.4088 & 1.5122 & 1.4977 & 0.84389 \\
\hline SD of Spread & 0.074574 & 0.014647 & 0.08599 & 0.062455 \\
\hline \multicolumn{5}{|l|}{ CF1 } \\
\hline Avg. Spread & 1.3621 & 1.517 & 1.4734 & 0.78473 \\
\hline SD of Spread & 0.022836 & 0.040547 & 0.14169 & 0.065962 \\
\hline \multicolumn{5}{|l|}{ CONSTR } \\
\hline Avg. Spread & 1.1651 & 1.2998 & 1.1346 & 0.9405 \\
\hline SD of Spread & 0.048443 & 0.071313 & 0.06642 & 0.064704 \\
\hline \multicolumn{5}{|l|}{ OSY } \\
\hline Avg. Spread & 0.93818 & 1.2396 & 1.2547 & 0.92908 \\
\hline SD of Spread & 0.037576 & 0.10688 & 0.027483 & 0.034359 \\
\hline
\end{tabular}

Table 8

Results of the multiobjective algorithm (using spacing) on the constrained test functions

\begin{tabular}{|c|c|c|c|c|}
\hline & MOMVO & MOALO & MODA & MOIMO \\
\hline \multicolumn{5}{|l|}{ BEL } \\
\hline Avg. Spacing & 0.0039105 & 0.0051075 & 0.010523 & 0.012261 \\
\hline SD of Spacing & 0.0033895 & 0.0043164 & 0.021808 & 0.016845 \\
\hline \multicolumn{5}{|l|}{$\mathrm{BNH}$} \\
\hline Avg. Spacing & 8.482 & 3.9153 & 4.2928 & 19.4547 \\
\hline SD of Spacing & 5.4558 & 2.3859 & 2.8649 & 2.3896 \\
\hline \multicolumn{5}{|l|}{$\mathrm{CF} 1$} \\
\hline Avg. Spacing & 0.014848 & 0.0037717 & 0.0035515 & 0.0046881 \\
\hline SD of Spacing & 0.024059 & 0.0026615 & 0.0021676 & 0.0018632 \\
\hline \multicolumn{5}{|l|}{ CONSTR } \\
\hline Avg. Spacing & 0.78491 & 0.46369 & 0.76356 & 0.95727 \\
\hline SD of Spacing & 0.2387 & 0.21466 & 0.30439 & 0.46124 \\
\hline \multicolumn{5}{|l|}{ OSY } \\
\hline Avg. Spacing & 22.9733 & 5.2117 & 9.9368 & 31.2487 \\
\hline SD of Spacing & 5.8054 & 3.2005 & 5.6156 & 5.9582 \\
\hline
\end{tabular}

\subsection{Engineering Benchmark Design Problems}

The final set of benchmark functions is the most stimulating one comprising six real-world engineering design problems. These problems have varied constrained characteristics. Table 9 to Table 12 present a quantitative comparison of all the four algorithms for different performance indicators. The results in these tables are in line with those in the preceding sections, in which the MOIMO approach generally presented superior convergence and coverage. Because of the difficulty of these benchmark problems, the better results exceedingly sustenance the dominance of the MOIMO approach and its applicability.

Fig. 4 presents the best Pareto optimal fronts for real-world engineering design problems. It may be seen in this figure that MOIMO has better qualitative characteristics amongst all algorithms despite multimodal search space and the existence of various limitations.

Table 9 
Outcomes of the multiobjective algorithm (GD) on the engineering design functions

\begin{tabular}{|c|c|c|c|c|}
\hline & MOMVO & MOALO & MODA & MOIMO \\
\hline \multicolumn{5}{|c|}{ Design of 3-Bar Truss } \\
\hline Avg. GD & 0.00081641 & 0.0014405 & 0.0011318 & 0.00062503 \\
\hline SD of GD & 0.00055249 & 0.0010217 & 0.00050327 & 0.0004354 \\
\hline \multicolumn{5}{|c|}{ Brushless DC Machine Design Problem } \\
\hline Avg. GD & 0.0013737 & 0.0018788 & 0.0043479 & 0.0027073 \\
\hline SD of GD & 0.0013662 & 0.0005236 & 0.0011936 & 0.00062814 \\
\hline \multicolumn{5}{|c|}{ Pressure Vessel Design } \\
\hline Avg. GD & 0.00154 & 0.0088965 & 0.0036704 & 0.0011863 \\
\hline SD of GD & 0.00016098 & 0.004002 & 0.00096662 & 0.0001821 \\
\hline \multicolumn{5}{|c|}{ Satellite Pipe Design } \\
\hline Avg. GD & 0.00060649 & 0.0010924 & 0.0032735 & 0.00063076 \\
\hline SD of GD & 0.00030408 & 0.00054764 & 0.0028936 & 0.00016805 \\
\hline \multicolumn{5}{|c|}{ Welded Beam Design Problem } \\
\hline Avg. GD & 0.0049408 & 0.0083299 & 0.0018358 & 0.0030226 \\
\hline SD of GD & 0.0039537 & 0.0062062 & 0.00097582 & 0.00098187 \\
\hline \multicolumn{5}{|c|}{ Car Crash Design Problem } \\
\hline Avg. GD & 0.0041605 & 0.076208 & 0.074507 & 0.059254 \\
\hline SD of GD & 0.0015295 & 0.033869 & 0.0065088 & 0.018628 \\
\hline
\end{tabular}
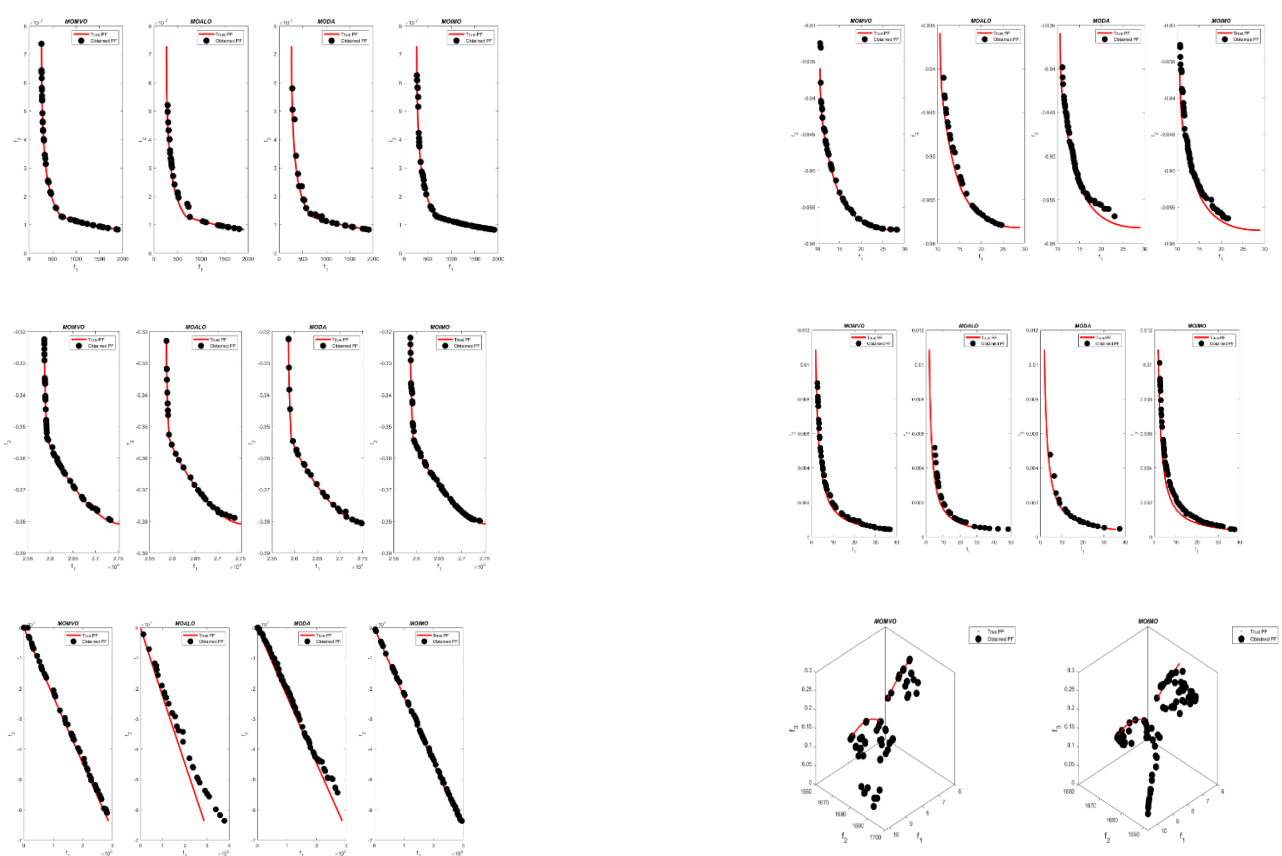

Fig. 4. Best Pareto front for real-world engineering benchmark functions

Table 10

Outcomes of the multiobjective algorithm (IGD) on the engineering design functions

\begin{tabular}{|c|c|c|c|c|}
\hline & MOMVO & MOALO & MODA & MOIMO \\
\hline \multicolumn{5}{|c|}{3 Bar Truss Design Problem } \\
\hline Avg. IGD & 0.00081641 & 0.0014405 & 0.0011318 & 0.00062503 \\
\hline SD of IGD & 0.00055249 & 0.0010217 & 0.00050327 & 0.0004354 \\
\hline \multicolumn{5}{|c|}{ Brushless DC Machine Design Problem } \\
\hline Avg. IGD & 0.0013737 & 0.0018788 & 0.0043479 & 0.0027073 \\
\hline SD of IGD & 0.0013662 & 0.0005236 & 0.0011936 & 0.00062814 \\
\hline \multicolumn{5}{|c|}{ Pressure Vessel Design } \\
\hline Avg. IGD & 0.00047789 & 0.0014181 & 0.00074622 & 0.00037128 \\
\hline SD of IGD & $3.914 \mathrm{e}-05$ & 0.00043574 & 0.00016207 & $6.4527 \mathrm{e}-05$ \\
\hline \multicolumn{5}{|c|}{ Satellite Pipe Design } \\
\hline Avg. IGD & 0.00048671 & 0.00072714 & 0.00083374 & 0.00067951 \\
\hline SD of IGD & 0.00015372 & 0.00014836 & 0.00015485 & 0.00046841 \\
\hline \multicolumn{5}{|c|}{ Welded Beam Design Problem } \\
\hline Avg. IGD & 0.0055088 & 0.010773 & 0.014105 & 0.0044458 \\
\hline SD of IGD & 0.003133 & 0.0039621 & 0.0062055 & 0.0030843 \\
\hline \multicolumn{5}{|c|}{ Car Crash Design Problem } \\
\hline Avg. IGD & 0.0041605 & 0.0080252 & 0.005071 & 0.0021731 \\
\hline SD of IGD & 0.0015295 & 0.0059468 & 0.0014011 & 0.00064515 \\
\hline
\end{tabular}

Table 11 
Outcomes of the multiobjective algorithm (Spread) on the engineering design functions

\begin{tabular}{|c|c|c|c|c|}
\hline & MOMVO & MOALO & MODA & MOIMO \\
\hline \multicolumn{5}{|c|}{3 Bar Truss Design Problem } \\
\hline Avg. Spread & 1.2768 & 1.3753 & 1.4348 & 0.99958 \\
\hline SD of Spread & 0.063482 & 0.06986 & 0.081976 & 0.037465 \\
\hline \multicolumn{5}{|c|}{ Brushless DC Machine Design Problem } \\
\hline Avg. Spread & 1.0505 & 1.1656 & 0.89866 & 0.88668 \\
\hline SD of Spread & 0.069106 & 0.096319 & 0.087243 & 0.065443 \\
\hline \multicolumn{5}{|c|}{ Pressure Vessel Design } \\
\hline Avg. Spread & 0.99371 & 1.3947 & 0.73348 & 0.7553 \\
\hline SD of Spread & 0.032797 & 0.057863 & 0.063679 & 0.05195 \\
\hline \multicolumn{5}{|c|}{ Satellite Pipe Design } \\
\hline Avg. Spread & 1.0261 & 1.2965 & 1.4348 & 0.90803 \\
\hline SD of Spread & 0.055868 & 0.10855 & 0.068078 & 0.034882 \\
\hline \multicolumn{5}{|c|}{ Welded Beam Design Problem } \\
\hline Avg. Spread & 1.0644 & 1.322 & 1.2264 & 0.78972 \\
\hline SD of Spread & 0.10156 & 0.047291 & 0.22633 & 0.019262 \\
\hline \multicolumn{5}{|c|}{ Car Crash Design Problem } \\
\hline Avg. Spread & 1.0185 & 1.2545 & 1.1225 & 0.79357 \\
\hline SD of Spread & 0.073509 & 0.090501 & 0.13589 & 0.093567 \\
\hline
\end{tabular}

Table 12

Outcomes of the multiobjective algorithm (Spacing) on the engineering design functions

\begin{tabular}{|c|c|c|c|c|}
\hline & MOMVO & MOALO & MODA & MOIMO \\
\hline \multicolumn{5}{|c|}{3 Bar Truss Design Problem } \\
\hline Avg. Spacing & 161.0758 & 48.0183 & 134.1771 & 409.7276 \\
\hline SD of Spacing & 40.4036 & 24.9562 & 62.3906 & 104.2308 \\
\hline \multicolumn{5}{|c|}{ Brushless DC Machine Design Problem } \\
\hline Avg. Spacing & 1.5504 & 0.58266 & 1.0003 & 1.545 \\
\hline SD of Spacing & 0.3786 & 0.19288 & 0.74956 & 0.36674 \\
\hline \multicolumn{5}{|c|}{ Pressure Vessel Design } \\
\hline Avg. Spacing & 8346712.3552 & 2309040.2674 & 8599005.8531 & 9895393.2942 \\
\hline SD of Spacing & 2829417.1318 & 385083.5572 & 2754131.991 & 1831215.4646 \\
\hline \multicolumn{5}{|c|}{ Satellite Pipe Design } \\
\hline Avg. Spacing & 209.9238 & 109.1347 & 87.9714 & 270.4866 \\
\hline SD of Spacing & 71.5969 & 64.8868 & 57.9485 & 75.78 \\
\hline \multicolumn{5}{|c|}{ Welded Beam Design Problem } \\
\hline Avg. Spacing & 5.7868 & 2.7399 & 2.0075 & 6.5448 \\
\hline SD of Spacing & 1.6432 & 1.4783 & 2.1302 & 2.7005 \\
\hline \multicolumn{5}{|c|}{ Car Crash Design Problem } \\
\hline Avg. Spacing & 2.9978 & 1.4769 & 4.0722 & 5.5785 \\
\hline SD of Spacing & 0.61387 & 0.92514 & 0.40501 & 1.0367 \\
\hline
\end{tabular}

Fig. 5 presents the overall performance comparison of all the algorithms in terms of best values of performance indicators and its (their) standard deviation over 10 independent runs. MOIMO obtains the best value of average GD and IGD for 8 and 13 times, respectively. GD and IGD are indicators of proximity to the true Pareto front. For 17 instances, MOIMO obtains the best value of spread presenting better coverage and convergence in all algorithms. MoS reflects the coverage of solutions. For 14 cases, MOIMO gets the best values of MoS. Except for MoS, for all three performance indicators, MOIMO presents minimum standard deviation proving the consistency of results. Thus, we can conclude that MOIMO provides the best results across all the algorithms for the majority of performance indicators.

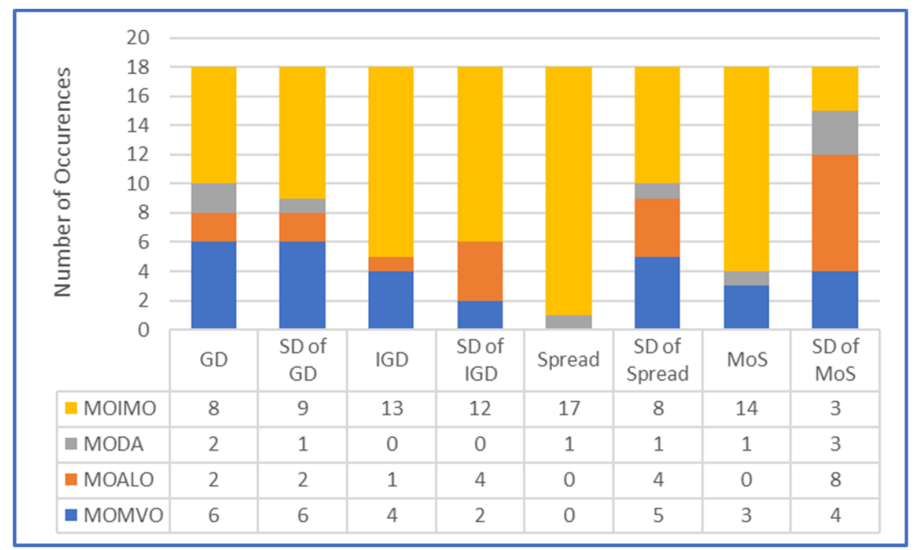

Fig. 5. Overall performance comparison of all the algorithms for benchmark test functions

To sum up, the outcomes demonstrate that MOIMO is highly capable and modest compared to the existing well-regarded approaches. High coverage and convergence can be two key reasons behind it. Higher convergence is the result of leader 
selection strategy wherein candidate solutions update their position considering the best non-dominated solution. High coverage is the result of storage maintenance and leader selection strategy. Since the leader is selected from the least populated region and solutions are removed from the dense area, the MOIMO has better coverage and diversity in all objectives. Despite all these merits, the present version of MOIMO can be applied to maximum four objectives. With an increasing number of objectives, MOIMO becomes less effective. This is because, for problems with more than four objectives, the archive gets full very quickly on account of a considerable number of non-dominated solutions. Besides, this approach is appropriate only for an objective function with a continuous variable and requires necessary changes for application on problems with discrete variables. The findings evidenced that MOIMO can be very useful for optimizing multiobjective problems. The MOIMO approach presented better coverage (diversification) and convergence (intensification). The superior intensification of MOIMO is owing to the updating solutions about the best non-dominated solutions attained up to now. The solutions move towards the best solutions. Also, the high convergence initiates from the adaptive mechanism, which quickens the travels of ions to the best non-dominated solutions obtained up to now in the storage. The storage maintenance and leader selection strategy result in higher coverage. It is worth stating here that as the updating mechanism of the ions in MOIMO is like IMO, MOIMO receives good exploration, local solutions evasion, fast convergence, and exploitation from this algorithm. Apart from convergence and coverage, algorithms are compared in terms of computational time in Table 13. For five different instances, MOIMO takes minimum simulation time, while for 12 examples, it stands second closely following the MOMVO. Thus, in terms of computational time, MOIMO presents excellent performance.

Table 13

Comparison of algorithms in terms of computational time

\begin{tabular}{|c|c|c|c|c|c|}
\hline \multirow[t]{2}{*}{ Sr. No. } & \multirow[t]{2}{*}{ Benchmark test function } & \multicolumn{4}{|c|}{ Algorithms } \\
\hline & & MOMVO & MOALO & MODA & MOIMO \\
\hline 1 & ZDT1 & 12.6218 & 15.350 & 63.934 & 16.993 \\
\hline 2 & ZDT2 & 1.889 & 2.5875 & 10.381 & 2.3625 \\
\hline 3 & ZDT3 & 0.9187 & 3.0265 & 15.073 & 1.545 \\
\hline 4 & ZDT4 & 0.6656 & 1.8812 & 5.6312 & 1.2562 \\
\hline 5 & ZDT6 & 1.215 & 2.484 & 11.435 & 2.0421 \\
\hline 6 & SCH1 & 2.5593 & 3.0031 & 4.7359 & 1.9218 \\
\hline 7 & $\mathrm{SCH} 2$ & 3.843 & 3.9687 & 7.6031 & 2.8219 \\
\hline 8 & BEL & 2.1125 & 2.9188 & 11.9688 & 2.3688 \\
\hline 9 & $\mathrm{BNH}$ & 4.3250 & 5.7266 & 16.5813 & 4.6016 \\
\hline 10 & CF1 & 4.9531 & 7.7188 & 21.8906 & 5.3438 \\
\hline 11 & CONSTR & 4.4063 & 7.0156 & 17.1875 & 3.4531 \\
\hline 12 & OSY & 2.375 & 5.0313 & 38.875 & 1.7813 \\
\hline 13 & 3-bar truss design & 11.875 & 12.5938 & 47.7188 & 11.9375 \\
\hline 14 & Brushless DC motor design & 15.7656 & 28.1094 & 108.2813 & 7.8125 \\
\hline 15 & Car crash design & 8.9219 & 13.3750 & 67.9531 & 10.2031 \\
\hline 16 & Pressure vessel design & 11.0469 & 23.8125 & 86.4531 & 14.4844 \\
\hline 17 & Satellite pipe design & 20.2344 & 28.7656 & 180.4063 & 15.1563 \\
\hline 18 & Welded beam design & 6.5 & 14.7344 & 121.8438 & 6.8281 \\
\hline
\end{tabular}

\section{Conclusion}

This effort projected a physics-inspired multiobjective approach is imitating the interaction of ions. A storage and leader selection strategy were then combined into a single objective IMO approach to solving multiobjective problems. A group of unconstrained, constrained and engineering benchmark functions were employed to assess the performance of the MOIMO. The outcomes of MOIMO are compared with those of MODA, MOMVO and MOALO. It was evident that the MOIMO algorithm is very competent and modest in determining an exact estimation of Pareto optimal front with uniform dispersal across all objectives with minimum simulation time.

Furthermore, the high convergence of MOIMO results in precisely estimated solutions and the uniform distribution is owing to the excellent exploration. Also, leader selection and storage preservation endorse the spreading of solutions. Examination of various constraint handling methods to solve MOOP using MOIMO can be an excellent future work. Also, the proposed approach can be applied to solving real-world problems.

\section{Acknowledgment}

The authors would like to thank Professor Seyedali Mirjalili and Shri Pradeep Jangir for their valuable support. 


\section{References}

Akbari, R., Hedayatzadeh, R., Ziarati, K., \& Hassanizadeh, B. (2012). A multi-objective artificial bee colony algorithm. Swarm and Evolutionary Computation, 2, 39-52.

Buch, H., \& Trivedi, I. (2020). A new non-dominated sorting ions motion algorithm: Development and applications. Decision Science Letters, 9(1), 59-76.

Coello, C. A. C. (2011). An introduction to multi-objective particle swarm optimizers. In Soft computing in industrial applications (pp. 3-12). Springer, Berlin, Heidelberg.

Coello, C. A. C., \& Cortés, N. C. (2005). Solving multiobjective optimization problems using an artificial immune system. Genetic Programming and Evolvable Machines, 6(2), 163-190.

Coello, C. C., \& Lechuga, M. S. (2002, May). MOPSO: A proposal for multiple objective particle swarm optimization. In Proceedings of the 2002 Congress on Evolutionary Computation. CEC'02 (Cat. No. 02TH8600) (Vol. 2, pp. 1051-1056). IEEE.

Coello, C. A. C. (2000). Use of a self-adaptive penalty approach for engineering optimization problems. Computers in Industry, 41(2), 113-127.

Coello, C. A. C. (2009). Evolutionary multi-objective optimization: some current research trends and topics that remain to be explored. Frontiers of Computer Science in China, 3(1), 18-30.

Deb, K. (2001). Multi-objective optimization using evolutionary algorithms (Vol. 16). John Wiley \& Sons.

Deb, Kalyanmoy, Samir Agrawal, Amrit Pratap, and T Meyarivan. 2000. "A Fast Elitist Non-Dominated Sorting Genetic Algorithm for Multi-Objective Optimization: NSGA-II.” In Parallel Problem Solving from Nature PPSN VI, eds. Marc Schoenauer et al. Berlin, Heidelberg: Springer Berlin Heidelberg, 849-58.

Dorigo, M., \& Di Caro, G. (1999, July). Ant colony optimization: a new meta-heuristic. In Proceedings of the 1999 congress on evolutionary computation-CEC99 (Cat. No. 99TH8406) (Vol. 2, pp. 1470-1477). IEEE.

Javidy, B., Hatamlou, A., \& Mirjalili, S. (2015). Ions motion algorithm for solving optimization problems. Applied Soft Computing, 32, 72-79.

Knowles, J., \& Corne, D. (1999, July). The pareto archived evolution strategy: A new baseline algorithm for pareto multiobjective optimisation. In Proceedings of the 1999 Congress on Evolutionary Computation-CEC99 (Cat. No. 99TH8406) (Vol. 1, pp. 98-105). IEEE.

Mirjalili, S. (2016). Dragonfly algorithm: a new meta-heuristic optimization technique for solving single-objective, discrete, and multi-objective problems. Neural Computing and Applications, 27(4), 1053-1073.

Mirjalili, S., Jangir, P., Mirjalili, S. Z., Saremi, S., \& Trivedi, I. N. (2017). Optimization of problems with multiple objectives using the multi-verse optimization algorithm. Knowledge-Based Systems, 134, 50-71.

Mirjalili, S., Gandomi, A. H., Mirjalili, S. Z., Saremi, S., Faris, H., \& Mirjalili, S. M. (2017). Salp Swarm Algorithm: A bio-inspired optimizer for engineering design problems. Advances in Engineering Software, 114, 163-191.

Mirjalili, S., Jangir, P., \& Saremi, S. (2017). Multi-objective ant lion optimizer: a multi-objective optimization algorithm for solving engineering problems. Applied Intelligence, 46(1), 79-95.

Mirjalili, S., Mirjalili, S. M., \& Hatamlou, A. (2016). Multi-verse optimizer: a nature-inspired algorithm for global optimization. Neural Computing and Applications, 27(2), 495-513.

Mirjalili, S., Saremi, S., Mirjalili, S. M., \& Coelho, L. D. S. (2016). Multi-objective grey wolf optimizer: a novel algorithm for multi-criterion optimization. Expert Systems with Applications, 47, 106-119.

Mirjalili, S. Z., Mirjalili, S., Saremi, S., Faris, H., \& Aljarah, I. (2018). Grasshopper optimization algorithm for multiobjective optimization problems. Applied Intelligence, 48(4), 805-820.

Ngatchou, P., Zarei, A., \& El-Sharkawi, A. (2005, November). Pareto multi objective optimization. In Proceedings of the 13th International Conference on, Intelligent Systems Application to Power Systems (pp. 84-91). IEEE.

Schott, J. R. (1995). Fault tolerant design using single and multicriteria genetic algorithm optimization (No. AFIT/CI/CIA95-039). Air Force Inst of Tech Wright-Patterson AFB OH.

Srinivas, N., \& Deb, K. (1994). Muiltiobjective optimization using nondominated sorting in genetic algorithms. Evolutionary computation, 2(3), 221-248.

Van Veldhuizen, D. A. (1999). Multiobjective Evolutionary Algorithms: Classifications, Analyses and New Innovations. IRE Transactions on Education.

Nanda, S. J. (2016, September). Multi-objective moth flame optimization. In 2016 International conference on Advances in computing, communications and informatics (ICACCI) (pp. 2470-2476). IEEE.

Wolpert, D. H., \& Macready, W. G. (1997). No free lunch theorems for optimization. IEEE transactions on evolutionary computation, 1(1), 67-82

Zhang, M., Wang, H., Cui, Z., \& Chen, J. (2018). Hybrid multi-objective cuckoo search with dynamical local search. Memetic Computing, 10(2), 199-208.

Zou, F., Wang, L., Hei, X., Chen, D., \& Wang, B. (2013). Multi-objective optimization using teaching-learning-based optimization algorithm. Engineering Applications of Artificial Intelligence, 26(4), 1291-1300. 


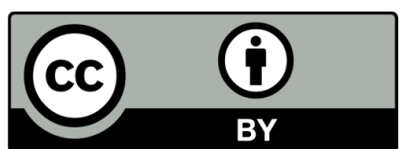

(C) 2021 by the authors; licensee Growing Science, Canada. This is an open access article distributed under the terms and conditions of the Creative Commons Attribution (CC-BY) license (http://creativecommons.org/licenses/by/4.0/). 